\title{
Prefacio / Preface
}

La Comunidad de Madrid, como el resto del Planeta, está sumida en la denominada «Crisis de la Biodiversidad». El resultado tangible de esta crisis es la pérdida rápida de organismos, tanto a nivel de especie como de poblaciones. Este problema es particularmente serio en una Comunidad como Madrid en la que se combinan un importante desarrollo industrial, un crecimiento espectacular de la superficie urbanizada y una gran expansión de infraestructuras viarias.

En estos aspectos Madrid es una región europea más, en rápida expansión económica, cuyo desarrollo pasa una alta factura a la Naturaleza. Sin embargo, se trata de un caso diferente al de otras zonas de Europa, ya que en Madrid el conocimiento de nuestra diversidad biológica es únicamente parcial. La última revisión de conjunto la realizó entre 1851 y 1858 el insigne Mariano de la Paz Graells, director del Museo Nacional de Ciencias Naturales, y desde entonces no se ha vuelto a abordar el problema. Y estamos hablando de la diversidad faunística, bien conocida en el resto de países de nuestro ámbito desde el siglo pasado, pero que por razones históricas y económicas nunca se estudió adecuadamente en nuestro país. Y para darse cuenta de esto basta con echar un vistazo a la literatura científica reciente. En los últimos cinco años se han añadido tres especies de anfibios nativos al listado de especies presentes en Madrid (dos de ellas endemismos ibéricos amenazados), se han descrito cinco especies nuevas de coleópteros (algunos endémicos de Madrid), un género y especie nueva de himenópteros.... y así con muchos otros grupos. Esto se traduce en un desconocimiento real de la diversidad faunística madrileña sobre todo debido a la carencia de información taxonómica y biogeográfica suficiente sobre la mayoría de las especies. Esta ausencia de información ha imposibilitado la elaboración de inventarios fiables de especies y las consiguientes propuestas sobre el estado de conservación de las mismas. Por ejemplo, menos de un $0,4 \%$ del total de especies de insectos que probablemente habitan en Madrid aparecen en la Directiva 92/43/CEE o en el Catálogo Regional de especies amenazadas.

Pero a pesar de este desconocimiento generalizado, a nivel político se habla de «conservar la biodiversidad», se establecen Parques Regionales sin conocer qué especies habitan en ellos y se elaboran planes de gestión en los que áreas singulares desde el punto de vista de la Biodiversidad se quedan sin protección o regulación. $\mathrm{O}$ incluso se fumigan los bosques y los cultivos «alegremente» sin saber cuántos endemismos madrileños se están eliminando. Estos problemas no son una trivialidad y como ejemplo baste recordar el «revuelo» informativo (toda la prensa nacional y las televisiones) causado por el descubrimiento de una especie nueva de escarabajo (Tillus ibericus) en un área donde estaba prevista la construcción de un campo de golf y las posibles consecuencias económicas que pueda tener. $\mathrm{O}$ el impacto causado por la contaminación de las aguas del río Manzanares.

La Comunidad de Madrid financió el proyecto titulado Evaluación del conocimiento y del estado de conservación de la diversidad animal de Madrid (GR/AMB/0750/2004), de un año de duración (2005), en el cual un amplio equipo de investigadores del Museo Nacional de Ciencias Naturales abordó el estudio de las fases iniciciales del conocimiento de esta diversidad. Y en este número especial de Graellsia se recopila una buena parte de los trabajos resultantes de dicho proyecto.

Como primer paso ineludible para el estudio de la Biodiversidad es necesaria la elaboración de listados taxonómicos exhaustivos y actualizados de cada uno de los taxones, unos listados que no existen para la mayoría de los grupos faunísticos de Madrid. En este volumen se presentan listados (aislados o dentro de estudios más amplios) de grupos tan diversos como reptiles, anfibios, moluscos (gasterópodos, bivalvos), insectos (diversas familias de coleópteros, himenópteros 
parasitoides y productores de agallas, dípteros acuáticos, malófagos), crustáceos (instersticiales y cavernícolas), arácnidos (arañas, ácaros del medio acuático) y rotíferos.

En segundo lugar se incluyen una serie de trabajos que tienen como objeto conocer la distribución geográfica de las especies, tanto mediante la compilación de la información faunística existente como con la elaboración de modelos predictivos de distribución. Para el desarrollo de este objetivo se han utilizado tres niveles de obtención de datos. En un primer nivel se compila toda la información ya disponible en publicaciones dispersas sobre la distribución geográfica de los grupos taxonómicos en la Comunidad de Madrid. En un segundo nivel se revisa el material ya existente en las colecciones científicas. De esta forma, el análisis conjunto de estos dos niveles permite obtener una idea precisa sobre la distribución histórica de los organismos. En esta monografía se incluyen trabajos en los que se presentan relaciones faunísticas completas del material disponible en las colecciones del MNCN (reptiles, ejemplares tipo de insectos, colección de tejidos...). El tercer nivel incluye la realización de muestreos de campo específicos que permitan conocer qué es lo que realmente hay y, especialmente, evaluar comparativamente la existencia de cambios en las distribuciones. Este grupo de trabajos incluyen Atlas de distribución preliminares.

Con los datos obtenidos, se ha procedido a estimar la distribución potencial de cada una de las especies consideradas mediante diversos modelos que, teniendo en cuenta los datos de presencia y diversas variables ambientales manejadas mediante un Sistema de Información Geográfica, permiten predecir con relativa fiabilidad la distribución potencial de las especies en el área de estudio, como es el caso de los himenópteros de la familia Cynipidae, y de los coleópteros de las familias Geotrupidae y Aphodiidae.

Una vez estimada la distribución individual de las distintas especies de los grupos seleccionados, se elaboran los listados de especies singulares de alto valor, tanto en función de su grado de rareza como por su endemicidad o su diferenciación filogenética, de forma que se puede proponer una lista actualizada de taxones que necesitan protección y otra de taxones que necesitan mayor estudio. Es impensable que en un país moderno no se sepa qué especies amenazadas habitan en un punto determinado antes de, por ejemplo, proceder a una fumigación aérea como las que se realizan en muchas áreas de Madrid, y la ignorancia no exime de la responsabilidad administrativa que el futuro reclamará. En nuestro caso, se utiliza el grupo de las Aves para describir la distribución geográfica de la riqueza de especies. En el grupo de las Aves, la información disponible es muy superior a la existente en otros grupos faunísticos y por ello se pueden plantear objetivos como éste, situados a otro nivel de análisis. El objetivo último es explorar qué rasgos de la biología de las especies son responsables de su rareza y la propensión a presentar problemas de conservación. Objetivos secundarios son poder distinguir la rareza natural con bases ecológicas y biogeográficas de aquella debida a problemas de conservación por efecto humano, catalogar las especies de aves madrileñas de acuerdo a su estatus de conservación y desarrollar una metodología aplicable a escala regional y que pueda utilizarse también en otros taxa.

Por último, se incluye un listado de la información molecular disponible en GenBank para las especies de vertebrados de la Comunidad de Madrid, una herramienta útil para el desarrollo de futuros estudios poblacionales y filogeográficos.

Muchos de los grupos cuyo estudio se aborda en esta monografía constituyen componentes fundamentales de la diversidad del Planeta. Sin embargo, en la mayor parte de los casos no existen catálogos nacionales o son muy antiguos, muchos del siglo XIX. Algunos de los grupos incluidos en este proyecto son grupos hiperdiversos de artrópodos terrestres, como las arañas; la fauna ibérica de arañas es una de las más ricas de Europa, cuenta con más de 1.000 especies citadas, y con un elevado grado de endemismo, en torno al $18 \%$.

En el caso de los dípteros quironómidos, se han descrito cerca de 12.000 especies en el mundo, de las cuales 460 se habían citado en la península Ibérica hasta 1997 y 206 de ellas en los 
ríos de la Comunidad de Madrid. En el momento actual las cifras han ido aumentando y el número de citas ha de incrementarse aún más con el estudio de los sistemas leníticos. El conocimiento de este grupo resulta de gran interés por su importancia como aporte de biomasa asimilable por gran número de depredadores y por su papel como indicadores de la calidad de los ecosistemas acuáticos.

El grupo de los cinípidos, o avispas inductoras de agallas (superfamilia Cynipoidea), comprende unas 3.000 especies en todo el mundo, agrupadas en cinco familias. Una faceta adicional de interés de los cinípidos es que sus agallas, la mayor parte de formas variadas y estructuralmente complejas, constituyen "puntos calientes" ecológicos, ya que proporcionan la base vital para multitud de especies animales, principalmente otros insectos.

Todos los representantes de la familia Pteromalidae son parasitoides de estados inmaduros de otros insectos. Teniendo en cuenta la gran diversidad de insectos que son atacados por los pteromálidos, estos parasitoides son un enemigo potencial de muchas plagas agrícolas y forestales y pueden actuar como controladores biológicos de las mismas.

Los Mallophaga son insectos que viven sobre aves y mamíferos, desarrollando todo su ciclo biológico en el cuerpo del hospedador. Como ectoparásitos obligados están distribuidos por todo el mundo en la mayoría de las familias de aves y presentes en algunos mamíferos. Su especificidad parasitaria les convierte en referencia para estudios de biodiversidad en las aves y mamíferos hospedadores así como para estudios de coespeciación y ecología de dichos hospedadores.

Un caso particular es el de la fauna intersticial y subterránea asociada a los medios de agua dulce de la Comunidad de Madrid. Por fauna intersticial entendemos aquellos organismos que habitan los intersticios de las riberas inundadas y los fondos profundos de arroyos y ríos. Es una fauna que se ha subdividido, por criterios de tamaño, en una Microfauna Intersticial, formada sobre todo por nematodos, oligoquetos, gastrotricos, rotíferos y grupos relacionados, y una Macrofauna Intersticial, constituida por ácaros acuáticos y crustáceos, predominantemente. A semejanza de la fauna acuática epigea, la fauna intersticial no sólo es indicadora del estado de salud biológica de las aguas, sino que, en su composición diferencial, guarda la historia reciente de estos medios, historia que puede ser parcialmente recuperada. Como en los casos anteriores los estudios sobre esta fauna en Madrid han sido fragmentarios en el espacio y el tiempo.

En ocasiones, al desconocimiento hay que añadir una taxonomía extraordinariamente compleja y confusa. La mayor parte de los estudios sistemáticos de Unionoidea (Moluscos, Bivalvos) se han desarrollado tradicionalmente con caracteres conquiológicos, que en muchos casos son homoplásicos. La diversidad dentro de los géneros varía desde aquéllos que incluyen sólo una especie (Potomida) a los que engloban más de 10 (Unio). Sin embargo, para el género Unio se han descrito hasta cerca de 1.000 "razas" o morfotipos, de dudosa adscripción taxonómica, que se han considerado en ocasiones "especies incipientes". Además, destaca de modo especial la poca información que se tiene acerca de los bivalvos dulceacuícolas que habitan los ríos madrileños.

En cuanto a los niveles de endemicidad y a la selección de especies singulares indicamos que la aracnofauna de Madrid incluye más de 200 especies distribuidas en 36 familias. Casi un 10\% de las especies son endemismos ibéricos, algunos de ellos exclusivos de la Sierra de Guadarrrama. Entre los moluscos testáceos hay una especie emblemática, Helicella madritensis, con localidad tipo en Madrid, con escasísimos datos conocidos.

Hay que destacar el preocupante estado de conservación de algunos grupos (unionoideos, anfibios, peces). La desaparición generalizada de los unionoideos, por ejemplo, se debe fundamentalmente al deterioro de su hábitat (contaminación de las aguas, industrialización, desecación, regulación de los ríos...), que afecta tanto directamente a los bivalvos que allí habitan, como a los hospedadores de sus larvas (generalmente peces), imprescindibles para completar el ciclo vital de los moluscos. Las náyades presentan una estrategia reproductiva muy peculiar: después de incubar las larvas, denominadas gloquidios, en sus 
branquias, son emitidas al agua donde se adhieren a las aletas o branquias del pez hospedador; allí permanecen hasta completar la metamorfosis y alcanzar el estado juvenil. Así, la desaparición de peces hospedadores del medio imposibilita que se cierre el ciclo de reproducción del bivalvo.

El conjunto de trabajos que aquí se presentan, constituyen una primera aproximación al estudio de la Biodiversidad de Madrid, pero creemos que esta aproximación, seguida de los estudios filogeográficos pertinentes, representa lo que debería constituir la línea de actuación para el estudio integral de la Biodiversidad Regional y, sobre todo, permite abordar y enfocar de manera mucho más coherente los posibles problemas de las actuaciones futuras sobre la Naturaleza madrileña. 